J. Austral. Math. Soc. 20 (Series A) (1975), 332-347.

\title{
CHANGING THE SCALAR MULTIPLICATION ON A VECTOR LATTICE
}

\author{
PAUL CONRAD
}

(Received 13 June 1974)

Communicated by E. Strzelecki

\section{Introduction}

Throughout this paper only abelian $l$-groups will be considered and $G$ will denote an abelian $l$-group. $G$ is large in the $l$-group $H$ or $H$ is an essential extension of $G$ if $G$ is an $l$-subgroup of $H$ and for each $l$-ideal $L \neq 0$ of $H$ we have $L \cap G \neq 0$. A $v$-hull of $G$ is a minimal vector lattice that contains $G$ and is an essential extension of $G$. Each $G$ admits a $v$-hull (Conrad (1970)).We shall be interested in the following properties of $G$.

I. $G$ admits a scalar multiplication so that it is a vector lattice.

II. Any two scalar multiplications of $G$ are connected by an $l$-automorphism of $G$.

III. $G$ admits a unique $v$-hull.

Suppose that $G$ satisfies $I$ and let be a scalar multiplication for $G$. Then each $l$-automorphism $\phi$ determines a new scalar multiplication $\Phi$.

$$
r \Phi g=(r \cdot(g \phi)) \phi^{-1} \text { for each } r \in R \text { and } g \in G .
$$

Note that $\phi$ is a linear $l$-isomorphism of $(G, \Phi)$ onto $(G, \cdot)$ and so connects the two scalar multiplications. Thus if $G$ satisfies II then $G$ admits essentially only one scalar multiplication.

Two $l$-automorphisms $\alpha$ and $\beta$ of $G$ determine the same scalar multiplication.

if and only if $(r \cdot(g \alpha)) \alpha^{-1} \beta=r \cdot(g \beta)$ for all $r \in R$ and $g \in G$ if and only if $(r \cdot h) \alpha^{-1} \beta=r \cdot\left(h \alpha^{-1} \beta\right)$ for all $r \in R$ and $h \in G$ if and only if $\alpha^{-1} \beta$ is linear with respect to $\cdot$.

Now let $\mathscr{L}$ be the group of all $l$-automorphisms of $G$ and let $\mathscr{L}:=\{\alpha \in \mathscr{L} \mid \alpha$ is linear with respect to $\cdot\}$. If $G$ satisfies II then there exists a one to one map of the set of all scalar multiplications of $G$ onto the set of all left cosets of $\mathscr{L} \cdot$ in $\mathscr{L}$. 
We shall show that II is satisfied by a large class of vector lattices and that each $l$-group can be embedded in a vector lattice that satisfies II. Whether or not each $l$-group satisfies II remains an open and very difficult question, even for totally ordered vector lattices.

If $G$ is a vector lattice with respect to two scalar multiplications, then the $l$-ideals of $G$ are subspaces under both multiplications. In the unordered case there is no such preassigned set of subspaces and Example 5.1 shows that if $U \neq 0$ is an unordered real vector space then II is not satisfied.

An endomorphism $\alpha$ of an $l$-group $G$ is a p-endomorphism (or a polar preserving endomorphism) if

$$
x, y \in G \text { and } x \wedge y=0 \text { imply } x \alpha \wedge y=0 .
$$

The set $S$ of all $p$-endomorphisms of $G$ is a semiring. Thus the subring $\mathscr{P}(G)$ of the endomorphism ring of $G$ that is generated by $S$ is a directed po-ring with positive cone $S . \mathscr{P}(G)$ is called the ring of polar preserving endomorphisms of $G$. If $G$ is archimedean then $\mathscr{P}(G)$ is an archimedean $f$-ring (see Bigard and Keimel (1969) or Conrad and Diem (1971)). A subring of $\mathscr{P}(G)$ that contains the identity $e$ and is $o$-isomorphic to $R$ will be called a real subfield of $\mathscr{P}(G)$.

PROPOSITION. There is a natural one to one correspondence between the real subfields of $\mathscr{P}(G)$ and the scalar multiplications on $G$. In particular, $G$ satisfies I if and only if $\mathscr{P}(G)$ is a po real vector space.

Proof. If $(G, \cdot)$ is a vector lattice and $a \in R$ then define $\cdot a \in \mathscr{P}(G)$

$$
(\cdot a) g=a \cdot g \text { for all } g \in G \text {. }
$$

The map $a \rightarrow \cdot a$ is an $o$-isomorphism of $R$ onto a real subfield of $\mathscr{P}(G)$. Since the only automorphism of the field $R$ is the identity, distinct scalar multiplications of $G$ map onto distinct real subfields of $\mathscr{P}(G)$. Thus the map $\eta$ of $\cdot$ onto the real subfield $\cdot R$ is one to one.

Now let $D$ be a real subfield of $\mathscr{P}(G)$ and let $\pi$ be the $o$-isomorphism of $R$ onto $D$. For each $r \in R$ and $g \in G$ define

$$
r \cdot g=(\pi r) g .
$$

Then $(G, \cdot)$ is a vector lattice and $\cdot R=D$. Thus $\eta$ is a one to one map of the scalar multiplication of $G$ onto the real subfields of $\mathscr{P}(G)$.

Finally each real subfield of $\mathscr{P}(G)$ determines a scalar multiplication of $\mathscr{P}(G)$ so that it is a po real vector space. Thus $G$ satisfies $I$ if and only if $\mathscr{P}(G)$ contains a real subfield if and only if $\mathscr{P}(G)$ is a po real vector space.

Let $(G, \cdot)$ and $(G, *)$ be vector lattices and let $\alpha$ be a group automorphism of $G$. 
COROLlary. $\alpha$ is a linear map of $(G, \cdot)$ onto $(G, *)$ if and only if $\alpha \cdot R \alpha^{-1}={ }^{*} R$.

Proof. $(\rightarrow)$ For each $a \in R$ and $g \in G$

$$
(\alpha \cdot a) g=\alpha(\cdot a g)=\alpha(a \cdot g)=a *(\alpha g)=* a(\alpha g)=\left({ }^{*} a \alpha\right) g .
$$

Thus $\alpha^{\prime} a={ }^{*} a \alpha$ and hence $\alpha \cdot a \alpha^{-1}={ }^{*} a$. Therefore $\alpha \cdot R \alpha^{-1}={ }^{*} R$.

$(\leftarrow)$ The map $a \stackrel{\tau}{\rightarrow} \alpha \cdot a \alpha^{-1}$ is an isomorphism of $\cdot R$ onto ${ }^{*} R$, and since $R$ admits only one automorphism, $\tau$ is the $o$-isomorphism $a \rightarrow^{*} a$. Thus

$$
\alpha \cdot a \alpha^{-1}={ }^{*} a \text { or } \alpha^{\cdot} a={ }^{*} a \alpha \text { for all } a \in R .
$$

Thus for $a \in R$ and $g \in G$

$$
\alpha(a \cdot g)=\alpha(\cdot a g)=(\alpha \cdot a) g=\left({ }^{*} a \alpha\right) g={ }^{*} a(\alpha g)=a *(\alpha g) .
$$

Therefore any results we obtain about I or II for $G$ have applications to $\mathscr{P}(G)$ and conversely.

\section{Archimedean $l$-Groups}

Throughout this section let $G$ be an archimedean $l$-group. In Conrad (1970), it is shown that $G$ admits a unique $v$-hull $G^{v}$, and Bleier (1971) proves that $G^{v}$ is the smallest archimedean vector lattice that contains $G$. Thus $G$ satisfies III. Also $G$ satisfies II since it admits at most one scalar multiplication. For if $(G, \cdot)$ and $(G, *)$ are vector lattices then the identity automorphism of $G$ is linear (see Conrad (1970)).

Iwasawa (1943) showed that if $G$ is divisible and complete then $G$ satisfies I. Thus if $G$ is essentially closed then it satisfies I. If $G$ has a basis and is laterally complete then $G$ is a cardinal product $\Pi T_{\alpha}$ of archimedean $o$-groups $T_{\alpha}$ and hence $G$ satisfies $I$ if and only if each convex $o$-subgroup is $o$-isomorphic to $R$. If $G$ is a subdirect sum of integers then the Dedekind-MacNeille completion $G^{\wedge}$ of $G$ is a vector lattice if and only if each $0<g \in G$ is unbounded (see Conrad (1970)).

Proposition 1.1. G satisfies I if and only if each principal l-ideal $G(g)$ satisfies $\mathbf{I}$.

Proof. If $G$ satisfies $I$ each $l$-ideal is a subspace. Now $G \subseteq G^{v}$. Thus since $G^{v}$ is archimedean each $G(g)$ is a subspace of $G^{v}$ (see Conrad (1970)) and hence $G=\bigcup_{\in G g} G(g)$ is a subspace of $G^{v}$.

Now $\mathscr{P}(G)$ is an archimedean $f$-ring and hence squares are positive. Thus a subring $K$ of $\mathscr{P}(G)$ that is isomorphic to $R$ is a totally ordered subring of $\mathscr{P}(G)$ and hence a real subfield provided that $e \in K$.

Proposition 1.2. If $S$ is an archimedean f-ring with identity $e$ then there 
exists a largest o-subring of $S$ that contains $e$. In particular, $S$ contains at most one real subfield.

Proof. By Bernau's embedding theorem (Bernau (1965)) we may assume that $S$ is an $l$-subring of the ring $D(X)$ of almost finite continuous functions on a Stone space $X$ and $e$ is the identity for $D(X)$. Let $F$ be an $o$-subring of $S$ that contains $e$. Then $F$ consists of constant functions - for otherwise there exists $f \in F$ such that $0<f(x)<f(y)<\infty$ for some pair $x, y \in X$. Thus there are positive integers $m$ and $n$ such that

$$
n f(x)<m e<n f(y) .
$$

Therefore $n f$ and $m e$ are not comparable, a contradiction.

Corollary. An archimedean l-group $G$ satisfies $I$ if and only if the largest $o$-subring of $\mathscr{P}(G)$ that contains $e$ is a real subfield. Since $\mathscr{P}(G)$ contains at most one real subfield, $G$ admits at most one scalar multiplication.

THEOREM 1.3. An archimedean l-group $G$ contains a largest $l$-subgroup $H$ that is a vector lattice. $H$ is the largest subspace of $G^{v}$ contained in $G$ and $H$ is an l-characteristic subgroup of $G$.

Proor. If $A$ and $B$ are $l$-subgroups of $G$ and vector lattices then they are subspaces of $G^{v}$ (Conrad (1970)). We show that the $l$-subgroup $C$ of $G$ generated by $A$ and $B$ is also a subspace of $G^{v}$ and hence a vector lattice.

The group $A+B$ is a subspace of $G^{v}$ and if $c \in C$ then

$$
c=V_{X} \wedge_{Y} t_{x y}
$$

where the $t_{x y}$ belong to $A+B$ and $X$ and $Y$ are finite. Thus for $r \in R$

$$
r c=r\left(\vee \wedge t_{x y}\right)=\vee \wedge\left(r t_{x y}\right) \in C .
$$

Thus $G$ contains a largest $l$-subgroup $H$ that is a vector lattice and $H$ is a subspace of $G^{v}$. The above argument shows that if $D$ is a subspace of $G^{v}$ contained in $G$ then the $l$-subgroup of $G$ generated by $D$ is also a subspace of $G^{v}$. Thus $H$ is the largest subspace of $G^{v}$ contained in $G$.

Finally suppose that $\alpha$ is an $l$-automorphism of $G$, then $H \alpha$ is an $l$-subgroup of $G$ and a vector lattice (any $l$-homomorphism of a vector lattice into $G^{v}$ is necessarily linear). Therefore $H \alpha \subseteq H$.

REMARK. If $G$ is an arbitrary $l$-group and an $l$-subgroup of a vector lattice $K$ then the above proof shows that $G$ contains a largest $l$-subgroup $H$ that is also a subvector lattice of $K$, and $H$ is the largest subspace of $K$ contained in $G$. Example 5.9 shows that even if $G$ is a vector lattice in its own right it need not equal $H$. 
THEOREM 1.4. For an archimedean l-group $G$ the following are equivalent.

1) $G$ satisfies $I$.

2) Each principal l-ideal $G(g)$ satisfies $I$.

3) $\mathscr{P}(G)$ satisfies $I$.

4) The largest o-subring of $\mathscr{P}(G)$ is a real subfield.

5) $G$ is divisible and each cut in $Q^{+} e$ contains an element of $\mathscr{P}(G)$, where $e$ is the identity for $\mathscr{P}(G)$.

6) $G$ is divisible and for an arbitrary $0<g \in G$ each cut in $Q^{+} g$ contains an element of $G$.

Proof. We have shown 1$), 2), 3), 4$ ) are equivalent and clearly if $G$ satisfies I then it is divisible. So we shall assume that $G$ and hence $\mathscr{P}(G)$ are divisible.

If $0<g \in G$ then a cut in $Q^{+} g$ contains at most one element from $G$. For suppose that $a, b \in G$ belong to the cut. Then $a, b \in G(g)$. Let $M$ be a maximal $l$-ideal of $G(g)$. Modulo $M a$ and $b$ determine the same cut in $Q^{+} g$ and so $a \equiv b \bmod M$ for all such $M$. Thus $a=b$.

$(4 \rightarrow 5) . Q^{+} e \subseteq F \cong R$, where $F$ is the real $o$-subfield of $\mathscr{P}(G)$. Thus each cut in $Q^{+} e$ contains an element of $F$.

$(5 \rightarrow 6)$. Let $(L, U)$ be a cut in $Q^{+} g$. Then the corresponding cut $(\bar{L}, \bar{U})$ in $Q^{+} e$ contain a unique element $\alpha$ from $\mathscr{P}(G)$. Thus $g \alpha$ is contained in $(L, U)$.

$(6 \rightarrow 1)$. Let $a$ be the element in $R$ determined by the cut $(L, U)$ in $Q^{+}$and let $h$ be the element in $G$ contained in the corresponding cut $(\bar{L}, \bar{U})$ in $Q^{+} g$. Define $a g=h$. This determines a scalar multiplication on $G$ so that it is a vector lattice.

PROPOSITION 1.5. For a vector lattice $H$ the following are equivalent.

1) $H$ is archimedean.

2) The scalar multiplication on each l-subspace $S$ of $H$ is unique.

Proof. $(1 \rightarrow 2)$. If $S$ is a vector lattice then it must be a subspace of $H$ (Con$\operatorname{rad}(1970))$.

$(2 \rightarrow 1)$. If $H$ is not archimedean then there exists $0<b \ll a$ in $H$. The subspace $R a \oplus R b$ of $H$ is totally ordered and hence an $l$-subspace of $H$. Let $f$ be a homomorphism of $R a$ into $R b$ that is not linear and define

$$
\left(r_{1} a+r_{2} b\right) \tau=r_{1} a+f\left(r_{1} a\right)+r_{2} b .
$$

This is an $o$-automorphism of $R a \oplus R b$ that is not linear and so can be used to define a new scalar multiplication on $R a \oplus R b$, but this contradicts (2).

It is an open question whether or not (1) is equivalent to:

3) The scalar multiplication on $H$ is unique. If $H$ is totally ordered then a slight generalization of the above proof shows that (3) implies (1). 


\section{The $l$-Group $V(\Gamma, R)$}

Let $\Gamma$ be a po-set such that no incomparable elements have a lower boundusually called a root system. Let $V=V(\Gamma, R)$ be the set of all functions from $\Gamma$ into the reals whose support satisfies the ACC. A component $v_{\gamma}$ of $v \in V$ is maximal if $v_{\gamma} \neq 0$ and $v_{\alpha}=0$ for all $\gamma\langle\alpha \in \Gamma$. Define $v \in V$ to be positive if each maximal component is positive. Then $V$ is a vector lattice with respect to the natural addition and scalar multiplication (Conrad, Harvey and Holland (1963)).

Let $A$ be an $l$-subgroup of $V$.A $v$-isomorphism $\tau$ of $A$ into $V$ is an $l$-isomorphism such that for each $a \in A, a_{\alpha}$ is a maximal component of $a$ if and only if $(a \tau)_{\alpha}$ is a maximal component of $a \tau$.

LEMMA 2.1. Each v-isomorphism $\tau$ of $V$ into itself is epimorphic.

Proof. Consider $\theta<v \in V$ with a maximal component $v_{\alpha}$. There is an element $u \in V$ with support $\alpha$ for which $(u \tau)_{\alpha}=v_{\alpha}$ since any $o$-isomorphism of $R$ into $R$ must be a multiplication by a positive real and hence an epimorphism.

Thus $V \tau$ is order dense in $V$ and so $\tau$ preserves all infinite joins and intersections that exist in $V$ (Bernau (1966)). Now $V \tau$ is laterally complete (i.e. each disjoint subset of $V$ has a least upper bound) and so the join $w$ of all the $u \tau$ (one for each maximal component of $v$ ) belongs to $V \tau$ and is $a$-equivalent to $v$ ( $m w \geqq v$ and $n v \geqq w$ for some positive integers $m$ and $n$ ). Thus $V$ is an $a$-extension of the $a$-closed $l$-group $V \tau$ and so $V=V \tau$. For a proof that $V$ and hence $V \tau$ is $a$-closed see Conrad (1966).

Lemma 2.2. If $A$ is an l-subgroup of $V$ and $(A, *)$ is a vector lattice then the scalar multiplication * can be extended to $V$ so that $(V, *)$ is also a vector lattice.

Proof. There exists a linear $v$-isomorphism $\tau$ of $(A, *)$ into $V$ that can be extended to a $v$-isomorphism $\alpha$ of $V$ into $V$. For a proof of this see Conrad (1970) ( $\tau$ is determined by a Banaschewski map for real subspaces but they are also rational subspaces and so we get $\alpha$ ). Now by Lemma $2.1 \alpha$ is epimorphic. For $r \in R$ and $v \in V$ define

$$
r \# v=(r(v \alpha)) \alpha^{-1} \text {. }
$$

This is a scalar multiplication for $V$ and for $a \in A$ we have

$$
r \# a=(r(a \tau)) \alpha^{-1}=((r * a) \tau) \alpha^{-1}=((r * a) \alpha) \alpha^{-1}=r * a
$$

so \# extends $*$.

Remark. Example 5.4 shows that $A$ need not be a subspace of $V$.

An $n$-automorphism of $V$ is a $v$-automorphism that induces the identity on each $V^{\gamma} / V_{\gamma}$ where 


$$
\begin{gathered}
V^{\gamma}=\left\{v \in V \mid v_{\alpha}=0 \text { for all } \alpha>\gamma\right\}, \text { and } \\
V_{\gamma}=\left\{v \in V \mid v_{\alpha}=0 \text { for all } \alpha \geqq \gamma\right\} .
\end{gathered}
$$

THeORem 2.3. Each $n$-characteristic l-subgroup A of $V$ satisfies II; in fact any two scalar multiplications on $A$ are connected by an n-automorphism of $V$. $A$ satisfies $\mathrm{I}$ if and only if $A$ is a subspace of $V$.

Proof. Let $*$ be a scalar multiplication so that $(A, *)$ is a vector lattice. By Lemma $2.2 *$ can be extended to $V$. Thus (see Conrad (1970)) there exists a linear $v$-isomorphism $\alpha$ of $(V, *)$ into $V$ and by Lemma $2.1 \alpha$ is epimorphic. Now $V^{\gamma} \alpha=V^{\gamma}$ and $V_{\gamma} \alpha=V_{\gamma}$ so $\alpha$ induces an $o$-automorphism on each $V^{\gamma} / V_{\gamma}$. But $V^{\gamma} / V_{\gamma} \cong R$ and so these $o$-automorphisms are multiplications by positive reals. Let $\bar{\alpha}$ be the $v$-automorphisms of $V$ determined by these multiplications. Then $\alpha \bar{\alpha}^{-1}$ is a linear $n$-automorphism of $(V, *)$ onto $V$ and since $A$ is $n$-characteristic

$$
(A, *) \alpha \bar{\alpha}^{-1}=A \text {. }
$$

In particular, $A$ is a subspace of $V$.

COROLLARY I. Each l-group can be embedded in a vector lattice that satisfies II.

Proof. The main theorem in Conrad, Harvey and Holland (1963) asserts that each $l$-group can be embedded in a suitable $V(\Gamma, R)$.

\section{COROLlary II. Each l-ideal of V satisfies I and II.}

Proof. It suffices to show that if $\theta<v \in V$ then the principal $l$-ideal $V(v)$ generated by $v$ is $n$-characteristic. For each $l$-ideal of $V$ is the join of a directed set of principal $l$-ideals and hence is $n$-characteristic.

Let $\tau$ be an $n$-automorphism of $V$. Then clearly $v$ and $v \tau$ are a-equivalent and hence

$$
V(v) \tau=V(v \tau)=V(v) .
$$

COROLLARY III. Let $\left\{A_{\lambda} \mid \lambda \in \Lambda\right\}$ be a set of a-closed o-groups (that is, Hahn groups). Then the cardinal sum $\sum A_{\lambda}$ and the cardinal product $\amalg A_{\lambda}$ of the $A_{\lambda}$ satisfy I and II.

Proof. $\amalg A_{\lambda}=V(\Delta, R)$ when $\Delta$ is the join of the $\Gamma\left(A_{\lambda}\right)$ and $\boldsymbol{\Sigma} A_{\lambda}$ is an l-ideal of $\coprod A_{\lambda}$.

COROLlaRY IV. If $G$ is an $n$-characteristic l-subgroup of $V$ then any two real subfields of $\mathscr{P}(G)$ are conjugate by a p-automorphism of $G$.

ProOF. This follows from the theory in the introduction and the fact that an $n$-automorphism of $V$ is a $p$-automorphism. 
Let $N$ be the group of the $n$-automorphisms of $V$. If $*$ is a scalar multiplication of $V$ then Theorem 2.3 asserts that there exist $\alpha \in N$ such that

$$
(r v) \alpha=r *(v \alpha) \text { for all } r \in R \text { and } v \in V \text {. }
$$

Thus each scalar multiplication of $V$ is determined by an $\alpha \in N$ and the scalar multiplications of $V$ determined by $\alpha, \beta \in N$ agree if and only if $\alpha \beta^{-1}$ is linear.

Let $A$ be a vector lattice. Then we may assume that $A$ is an $l$-subspace of $V=V(\Gamma, R)$ for a suitable $\Gamma$. Suppose that $*$ is another scalar multiplication for $A$. Then we can extend $*$ to $V$ and there exists a linear $n$-automorphism $\tau$ of $(V, *)$ onto $V$. In particular, $A$ and $A \tau$ are subspace of $V$ and $r^{*} a=(r(a \tau)) \tau^{-1}$ for each $r \in R$ and $a \in A$. Conversely if $\tau$ is an $n$-automorphism of $V$ and $A \tau$ is a subspace of $V$ then for each $a \in A$ and $r \in R$ we define $r * a=(r(a \tau)) \tau^{-1}$. Then $(A, *)$ is a vector lattice and $\tau$ is a linear $l$-isomorphism of $(A, *)$ onto $A$.

Therefore the scalar multiplications of $A$ are determined by the n-automorphisms of $V$ that map A onto a subspace of $V$.

\section{The $l$-Group $\Sigma(\Gamma, R)$}

Let $V=V(\Gamma, R)$ be the vector lattice investigated in the last section. Let

$$
\begin{aligned}
\Sigma=\Sigma(\Gamma, R) & =\{v \in V \mid \text { support of } v \text { is finite }\} \\
F=F(\Gamma, R) & =\{v \in V \mid \text { support of } v \text { lies on a finite number of } \\
& \text { chains in } \Gamma\}
\end{aligned}
$$

A value of an element $g$ of an $l$-group $G$ is an $l$-ideal of $G$ that is maximal without containing $g . G$ is finite valued if each $g \in G$ has only a finite number of values. The set $\Gamma=\Gamma(G)$ of all the values of elements in $G$ is a root system.

In Conrad (1974) it is shown that if $A$ is a finite valued vector lattice with countable dimension then there exists a linear $l$-isomorphism of $A$ onto $\Sigma(\Gamma, R)$, where $\Gamma$ is the index set for the set of all the regular subgroups of the $l$-group $A$. In particular, $A$ is completely determined by the root system $\Gamma$.

THEOREM 3.1. If $A$ is a finite valued l-group then any two scalar multiplications of $A$ for which the dimension of $A$ is countable are connected by a $v$-automorphism of $A$.

Proor. Let $*$ and \# be two such scalar multiplications. Then $(A, *) \cong \Sigma(\Gamma, R)$ $\cong(A \#)$.

CoROLLARY. Let $\Gamma$ be a countable root system and let $\Sigma=\Sigma(\Gamma, R)$ with the natural scalar multiplication. If $*$ is a new scalar multiplication for $\Sigma$ then $\Sigma$ and $(\Sigma, *)$ are connected by a v-automorphism if an only if $(\Sigma, *)$ has countable dimension. 
REMARK. We have been unable to determine whether or not $(\Sigma, *)$ always has countable dimension. If so, then of course $\Sigma$ satisfies II.

THEOREM 3.2. Suppose that $G$ is a finite valued l-group and $\Gamma(G)$ satisfies the $D C C$.

1) $\Sigma(\Gamma, R)=F(\Gamma, R)$ is the unique a-closure of $G$.

2) $\Sigma(\Gamma, R)$ is the unique a-extension of $G$ that is a vector lattice.

3) $\Sigma(\Gamma, R)$ is the unique v-hull of $G$ that is also an a-extension.

Proof. Recall that $H$ is an $a$-extension of $G$ if $H$ is an $l$-group, $G$ is an $l$-subgroup if $H$ and each $0<h \in H$ is an $a$-equivalent to an element $0<g \in G$ or equivalently $L \rightarrow L \cap G$ is a one to one mapping of the set of $l$-ideals of $H$ onto the $l$-ideals of $G$. An a-closure of $G$ is an $a$-extension of $G$ that does not admit a proper $a$-extension. Each group admits an a-closure but usually not a unique one (Conrad (1966) or Wolfenstein).

We first show that each $a$-extension $H$ of $G$ is finite valued. Here we do not need the fact that $\Gamma$ satisfies the DCC. For $0<h \in H$ there is an element $0<g \in G$ such that $\eta g>h$ and $n h>g$ for some $n>0$. Let $\left\{H_{\lambda} \mid \lambda \in \Lambda\right\}$ be the set of all values of $h$ in $H$. Then they are also values of $g$. Thus $\left\{H_{\lambda} \cap G \mid \lambda \in \Lambda\right\}$ is a set of values of $g$ in $G$ and hence $\Lambda$ is finite.

(1) Let $K$ be an $a$-closure of $G$. Then since $K$ is finite valued, divisible and $\Gamma(K)$ satisfies the DCC there is a value preserving $l$-isomorphism $\sigma$ of $K$ such that

$$
K \sigma=\Sigma\left(\Gamma, K^{\gamma} / K_{\gamma}\right)
$$

where $K^{\gamma}$ is the intersection of all the $l$-ideals of $K$ that properly contain $K_{\gamma}$ (see Theorem 4.9 in Conrad (1970)). In particular, each $K^{\gamma} / K_{\gamma}$ is $o$-isomorphic to a subgroup $S_{\gamma}$ of $R$ and so there exists an $l$-isomorphism $\tau$ of $K$ so that

$$
K \tau=\Sigma\left(\Gamma, S_{\gamma}\right) \subseteq \Sigma(\Gamma, R) .
$$

But clearly $\Sigma(\Gamma, R)$ is an $a$-extension of $\Sigma\left(\Gamma, S_{\gamma}\right)$ and so since $K \tau$ is a-closed, $K \tau=\Sigma(\Gamma, R)$. Now $F(\Gamma, R)$ is always an a-closure of $\Sigma(\Gamma, R)$ (Conrad (1966) p. 147) and so in our case $F=\Sigma$.

(2) Suppose that $K$ is an $a$-extension of $G$ that is a vector lattice. Then each $K^{\gamma} / K_{\gamma} \cong R$ and so

$$
K \tau=\Sigma\left(\Gamma, S_{\gamma}\right)=\Sigma(\Gamma, R) .
$$

(3) Since a $v$-hull of $G$ is a vector lattice this is a special case of (2).

COROLlary. For a root system $\Gamma$ the following are equivalent.

a) $\Sigma(\Gamma, R)$ is a-closed.

b) $\Sigma(\Gamma, R)=F(\Gamma, R)$.

c) $\Gamma$ satisfies the DCC. 
Proof. We have shown c) $\rightarrow$ a) $\rightarrow$ b) and since $F$ is always $a$-closed $(b \rightarrow a)$. $(\mathrm{a} \rightarrow \mathrm{c})$ If $\gamma_{1}>\gamma_{2}>\cdots$ is an inversely well ordered descending chain in $\Gamma$ then let $a$ be the element in $V(\Gamma, R)$ such that

$$
a_{\gamma}=\left\{\begin{array}{ll}
1 & \text { if } \gamma=\gamma_{i} \\
0 & \text { otherwise. }
\end{array} \quad \text { for some } i\right.
$$

Then an easy computation shows that $[a] \oplus \Sigma$ is an $a$-extension of $\Sigma$ and hence the chain must be finite.

Remark. In Conrad (1970) it is shown that for a totally ordered group $G$ of finite rank a $v$-hull need not be an $a$-extension so $G$ need not have a unique $v$-hull. Example 5.5 shows that $\Sigma(\Gamma, R)$ need not be an $n$-characteristic subgroup of $V(\Gamma, R)$ so we cannot conclude from the theory in the last section that $\Sigma(\Gamma, R)$ satisfies II.

THEOREM 3.3. If $G$ is a finite valued l-group and $\Gamma(G)$ satisfies the DCC then the following are equivalent.

1) $G$ satisfies $I$.

2) Each $G^{\gamma} / G_{\gamma}$ is o-isomorphic to $R$.

3) $G \cong \Sigma(\Gamma, R)$.

4) $G$ is a-closed.

If this is the case then $G$ satisfies II.

Proof. $1 \rightarrow 2$, and $3 \rightarrow 1$ and 2 are obvious. By Theorem $3.2 \Sigma$ is the unique $a$-closure of $G$ and hence $3 \leftrightarrow 4$.

$(2 \rightarrow 3)$. Since each $G^{\gamma} / G_{\gamma}$ is divisible there exists a $v$-isomorphism $\sigma$ such that

$$
\Sigma\left(\Gamma, G^{\gamma} / G_{\gamma}\right) \subseteq G \sigma \subseteq V\left(\Gamma, G^{\gamma} / G_{\gamma}\right)
$$

(Conrad (1970)) and since $G^{\gamma} / G_{\gamma} \cong R$ for each $\gamma \in \Gamma$ we may assume

$$
\Sigma(\Gamma, R) \subseteq G \sigma \subseteq V(\Gamma, R) .
$$

Now $\Gamma$ satisfies the DCC and so by the proof of Theorem 4.9 in Conrad (1970) we have $\Sigma(\Gamma, R)=G \sigma$.

Now suppose that $*$ is another scalar multiplication for $\Sigma$ and for each $\gamma \in \Gamma$ define $e(\gamma)$

$$
e(\gamma)_{\alpha}= \begin{cases}1 & \text { if } \alpha=\gamma \\ 0 & \text { otherwise }\end{cases}
$$

Then $E=\{e(\gamma) \mid \gamma \in \Gamma\}$ is a basis for $\Sigma$ and an independent subset of $(\Sigma, *)$ so the identity map on $E$ can be lifted to linear $v$-isomorphism $\tau$ of $\Sigma$ into $(\Sigma, *)$. Since $\Sigma$ is $a$-closed and $(\Sigma, *)$ is an $a$-extension of $\Sigma \tau, \tau$ is epimorphic and hence $G$ satisfies II. 
THEOREM 3.4. If $G$ is an a-closed l-group that satisfies each bounded disjoint subset is finite,

Then $G$ satisfies I and II.

Proof. $F(\Gamma, R)$ is the unique $a$-closure of an $l$-group that satisfies (F) (see Conrad (1966)). Thus $G \cong F$ and so $G$ satisfies $I$.

In Conrad (1966) it is shown that if $G$ is a vector lattice that satisfies (F) then there exists a linear $v$-isomorphism $\tau$ such that $\Sigma(\Gamma, R) \subseteq G \tau \subseteq F(\Gamma, R)$ and hence $F$ is an $a$-extension of $G \tau$. Thus if $G$ is $a$-closed $G \tau=F$ and so II is satisfied.

REMARK. If $G$ satisfies (F) then $F(\Gamma, R)$ is an $l$-ideal of $V(\Gamma, R)$ and so Theorem 3.4 follows immediately from Corollary II of Theorem 2.3. Byrd (1966) gives an example that shows that in general $F(\Gamma, R)$ need not be $n$-characteristic in $V(\Gamma, R)$.

Note that if $\Gamma(G)$ is finite then $G$ satisfies the hypothesis of Theorem 3.2 and 3.3. Also if $G$ satisfies these hypotheses then any two real subfields of $\mathscr{P}(G)$ are conjugate by a $p$-automorphism of $G$.

\section{Totally Ordered Groups}

Throughout this section $G$ will denote a totally ordered group with $\Gamma$ the index set for the set of components $G^{\gamma} / G_{y}$ of $G . V(\Gamma, R)$ is the unique $a$-closure of $G$ (Hahn 1907)). Thus if $G$ is $a$-closed then $G \cong V$ and so by Theorem $2.3 G$ satisfies $I$ and II and so does each $l$-ideal of $V$. The next Proposition shows that this is all we can conclude from Theorem 2.3.

PROPOSITION 4.1. An n-characteristic subgroup $L$ of $V$ that is also a subspace is convex and conversely.

Proof. We show that $V(g) \subseteq L$ for each $0<g \in L$. Let $g_{\gamma}$ be the maximal component of $g$ and consider $0<h \in V(g)$. If $V(h) \neq V(g)$ then $h \ll g$ and o there exists an $n$-automorphism of $V$ that maps $g$ onto $g+h$. Then $h=g+h-g \in L$. If $V(h)=V(g)$ then there exists $r \in R$ such that $h_{\gamma}=r g_{\gamma}$ and so there exists an $n$-automorphism of $V$ that maps $r g$ onto $h$.

Note that we need the total order of $G$. For if $H=R \oplus R$ then $\{(x, x) \mid x \in R\}$ is an $n$-characteristic $l$-subgroup of $H$ and a subspace but it is not an $l$-ideal.

Now suppose that $\Gamma$ satisfies the DCC then $V=\Sigma(\Gamma, R)$ and by Theorems 3.1 and 3.2 we have:

$V$ is the unique $a$-extension of $G$ that is a vector lattice.

$V$ is the unique $v$-hull of $G$ that is also an $a$-extension. 
Moreover the following are equivalent: $G$ satisfies $\mathrm{I}$; each $G^{\gamma} / G_{\gamma} \cong R ; G \cong V$; $G$ is $a$-closed.

Proposition 4.2. For an o-group $G$ the following are equivalent.

1) G satisfies $I$.

2) Each $G(g)$ satisfies I.

Proof. $(1 \rightarrow 2)$ Clear.

$(2 \rightarrow 1)$ Let $S$ be the collection of all pairs $(L, *)$ where $L$ is a convex subgroup of $G$ and an ordered vector space with respect to the scalar multiplication * Define $(L, *) \leqq(H, \#)$ if $(L, *)$ is a subspace of $(H, \#)$. Then by Zorn's lemma there exists a maximal element $(M, *)$ in $S$. We show $M=G$. Suppose by way of contradiction that $0<g \in G \backslash M$. Then $G(g) \supset M$ and since $M$ is divisible $G(g)=M \oplus D$ a lexicographic extension of $M$ by the $o$-group $D$. Now by hypothesis $G(g)$ admits a scalar multiplication \# and since $M$ is contained in $G(g),(M, \#)$ is a subspace. Thus $G(g) / M \cong D$ is also an ordered vector spacesay $(D, O)$. For $r \in R$ and $m+t \in M \oplus D$ define

$$
r \cdot(m+d)=r * m+r \circ d .
$$

Then $(G(g), \cdot)$ is an ordered vector space and $(M, *)$ is a subspace, but this contradicts our choice of $M$ and so $M=G$ satisfies $I$.

\section{Examples and open questions}

EXAMPLE 5.1. A real non-ordered vector space $U$ does not satisfy II. For let $\alpha$ be a group isomorphism of $R$ onto the direct sum $\oplus_{\Lambda} R_{\lambda}$ and for $r$ in the field $R$ and $x$ in the group $R$ define

$$
r \circ x=(r(x \alpha)) \alpha^{-1}
$$

where $r(x \alpha)$ is the natural scalar multiplication in $\oplus_{\Lambda} R_{\lambda}$. Then $(R, 0)$ is a real vector space of dimension $|\Lambda|$. Thus if $|\Lambda|>1$ then $(R, \circ)$ and $(R, \cdot)$ are not connected by a group automorphism.

EXAMPLE 5.2. $R=D \oplus Q$ lexicographically ordered is a totally ordered group and a real vector space but it does not satisfy I. Also the cardinal sum $D \oplus Q$ is an archimedean $l$-group and a real vector space that does not satisfy $\mathrm{I}$.

EXAMPLE 5.3. Let $G$ be the subgroup of the cardinal product $\prod_{i=1}^{\infty} R_{i}$ generated by $\sum_{i=1}^{\infty} R_{i}$ and $(1,1,1, \cdots)$. Then $G$ is an $l$-group and each $G^{\gamma} / G_{\gamma} \cong R$ except $G / \Sigma R_{i}$, but $G$ does not satisfy I since it is not divisible.

If we totally order $\bigsqcup R_{i}$ by defining $\left(x_{1}, x_{2}, \cdots\right)$ to be positive if the first non-zero $x_{i}$ is positive, then $G$ is an $o$-group with each $G^{\gamma} / G_{\gamma} \cong R$ and $G / C$ satisfies I for each non-zero convex subgroup $C$ of $G$, but $G$ does not satisfy I. 
Let $H=\sum_{i=1}^{\infty} R_{i} \oplus Q(1,1,1, \cdots)$ the divisible hull of $G$. Then $G$ admits a scalar multiplication so that it is a real vector space, since its dimension as a rational vector space is large enough. If we impose the cardinal order on $H$ then it does not satisfy $I$; for then it would have to be a subspace of the vector lattice $\coprod_{i=1}^{\infty} R_{i}$.

One should be able to show that if we impose the above total order on $H$ then $H$ does not satisfy I. If $H$ does satisfy I then it follows from Lemma 2.2 and Theorem 2.3 that there exists an $n$-automorphism $\tau$ of $\bigsqcup R_{i}$ such that $H \tau$ is a subspace.

EXAMPLE 5.4. Let $V=\coprod_{i=0}^{\infty} R_{i}$ be totally ordered as in the last example. Let $\mu$ be a group isomorphism of $R$ onto $\coprod_{i=1}^{\infty} Q_{i}$

Define $\tau$

$$
a \rightarrow\left(\mu_{1}(a), \mu_{2}(a), \cdots\right) \text {. }
$$

$$
\left(a_{0}, a_{1}, a_{2}, \cdots\right) \tau=\left(a_{0}, \mu_{1}\left(a_{0}\right)+a_{1}, \mu_{2}\left(a_{0}\right)+a_{2}, \cdots\right) .
$$

Then $\tau$ is an $n$-automorphism of $V$. Now $A=\left(\sum_{i=0}^{\infty} R_{i}\right) \tau$ is $o$-isomorphic to $\sum_{i=0}^{\infty} R_{i}$ and so it admits a scalar multiplication but it is not a subspace of $V$. For pick the $a \in R$ for which $a \mu=(1,1,1, \cdots)$. Then $(a, 0,0, \cdots) \tau=(a, 1,1, \cdots) \in A$ but $r(a, 1,1, \cdots) \notin A$ for $r \in R \backslash Q$.

EXAMPLE 5.5. Let $V=\coprod_{i=1}^{\infty} R_{i}$ totally ordered as above and let $G=\sum_{i=1}^{\infty} R_{i}$. Then the map

$$
\begin{aligned}
& (1,0,0, \cdots) \rightarrow(1,1,1, \cdots) \\
& (0,1,0, \cdots) \rightarrow(0,1,1, \cdots)
\end{aligned}
$$

determines a linear $v$-isomorphism $\sigma$ of $\Sigma$ into $V$ such that

$$
\Sigma \subset \Sigma \sigma \subset V \text {. }
$$

The map

$$
\begin{aligned}
& (1,0,0, \cdots) \rightarrow(1,1,0,0, \cdots) \\
& (0,1,0, \cdots) \rightarrow(0,1,1,0, \cdots)
\end{aligned}
$$

determines a linear $v$-isomorphism of $\Sigma$ onto a proper subgroup of itself.

The map

$$
\begin{aligned}
& (1,0,0, \cdots) \rightarrow(1,1,1, \cdots) \\
& (0,1,0, \cdots) \rightarrow(0,1,0 \cdots) \\
& (0,0,1,0, \cdots) \rightarrow(0,0,1,0, \cdots)
\end{aligned}
$$


determines a map $\sigma$ of $\Sigma$ into $V$ such that $\Sigma|| \Sigma \sigma$.

EXAMPLE 5.6. Let

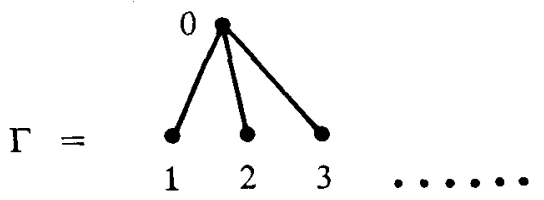

and let $V=V(\Gamma, R)$. The map

$$
\begin{aligned}
& (1,0,0, \cdots) \rightarrow(1,1,1, \cdots) \\
& (0,1,0, \cdots) \rightarrow(0,1,0, \cdots) \\
& (0,0,1,0, \cdots) \rightarrow(0,0,1,0, \cdots)
\end{aligned}
$$

determines an $n$-isomorphism $\sigma$ of $\Sigma$ into $V$ such that $\Sigma|| \Sigma \sigma$.

EXAMPLE 5.7. An $a$-closed archimedean $l$-group need not satisfy I. Let

$$
G=\prod_{i=1}^{\infty} Z_{i} \subset C \subset \prod_{i=1}^{\infty} R_{i}
$$

cardinally ordered, where $C$ consists of all the elements of the form $g+\left(x_{1}, x_{2}, \cdots\right)$ where $g \in G$ and $0 \leqq x_{i} \leqq 1$ and the number of distinct $x_{i}$ is finite. Thus $C=G+F$, where $F$ is the group of all elements in $\amalg R_{i}$ with finite range. It is shown in Con$\operatorname{rad}(1966)$ that $C$ is an $a$-closure of $G$. If $C$ is a vector lattice then it must be a subspace of $\prod R_{i}$, but $\sqrt{ } 2(1,2,3, \cdots) \notin C$.

Note also that the $v$-hull $G^{v}$ of $G$ is not an $a$-extension of $G$. For clearly $G^{v} \supset C$. Actually

$$
\begin{aligned}
G^{v}= & \left\{a \in \coprod R_{i} \mid \text { there exists reals } r_{1}, \cdots, r_{k} \text { such that each component of } a\right. \\
& \text { is of the form } \left.x_{1} r_{1}+x_{2} r_{2}+\cdots x_{k} r_{k} \text { with } x_{t} \in Z\right\} .
\end{aligned}
$$

REMARK. It can be shown that a hyper-archimedean $a$-closed $l$-group need not satisfy $I$.

EXAMPLE 5.8. A minimal vector lattice that contains the $o$-subgroup $[1] \oplus[\sqrt{ } 2] \oplus[\pi]$ of $R$ need not be totally ordered. Let $f$ be a homomorphism of $R$ into $R \oplus R ; f(a)=\left(f_{1}(a), f_{2}(a)\right)$ where

Let $\Gamma=\bigwedge_{1}^{0} \quad \begin{aligned} f(\pi) & =(\pi+1, \pi) \\ \text { and let } V & =V(\Gamma, R) .\end{aligned}$

$$
\begin{aligned}
& f(1)=(1,1) \\
& f(2)=(\sqrt{ } 2, \sqrt{ } 2+1) \\
& f(\pi)=(\pi+1, \pi) .
\end{aligned}
$$


Define $\left(a_{0}, a_{1}, a_{2}\right) \tau=\left(a_{0}, a_{1}+f_{1}\left(a_{0}\right), a_{2}+f_{2}\left(a_{0}\right)\right)$. Then $\tau$ is an $n$-automorphism of $V$.

Define $r *(x \tau)=(r x) \tau$ for all $x \in V$ and $r \in R$. Then $(V, *)$ is a vector lattice. $r *\left(a_{0}, a_{1}+f_{1}\left(a_{0}\right), a_{2}+f_{2}\left(a_{0}\right)\right)=\left(r a_{0}, r a_{1}, r a_{2}\right) \tau=\left(r a_{0}, r a_{1}+f_{1}\left(r a_{0}\right), r a_{2}+f_{2}\left(r a_{0}\right)\right)$. If $a_{0}=1$ and $a_{1}=a_{2}=-1$ we have

$$
r *(1,0,0)=\left(r,-r+f_{1}(r),-r+f_{2}(r)\right) .
$$

In particular

$$
\begin{aligned}
\sqrt{ } 2 *(1,0,0) & =(\sqrt{ } 2,0,1) \\
\pi *(1,0,0) & =(\pi, 1,0) .
\end{aligned}
$$

Now let $G$ be the $o$-subgroup of $V$ generated by $(1,0,0),(2,0,0)$ and $(\pi, 0,0)$. Then $V$ is a minimal vector lattice that contains $G$. Of course $V$ is not the $v$-hull of $G$.

EXAMPLE 5.9. A finite valued $l$-group $G$ with $\Gamma(G)$ satisfying the DCC that admits two non-isomorphic $v$-hulls. Let $\Gamma$ be the root system

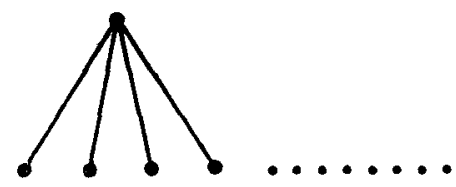

and let $V=V(\Gamma, R)$. Let $f$ be an isomorphism of $R$ onto $\coprod_{i=1}^{\infty} R_{i}$ such that $f(1)=(1,0,0, \cdots)$ and in general $f(x)=\left(f_{1}(x), f_{2}(x), \cdots\right)$. Define

$$
\left(x ; x_{1}, x_{2}, \cdots\right) \tau=\left(x ; x_{1}+f_{1}(x), x_{2}+f_{2}(x), \cdots\right) .
$$

Then $\tau$ is an $n$-automorphism of $V$. For $v \in V$ and $r \in R$ define $r \cdot(v \tau)=(r v) \tau$. Then $(V, \cdot)$ is a vector lattice.

$$
\begin{aligned}
r \cdot\left(x ; f_{1}(x), f_{2}(x), \cdots\right) & =r \cdot(x ; 0,0, \cdots) \tau=(r x ; 0,0, \cdots) \tau \\
& =\left(r x ; f_{1}(r x), f_{2}(r x), \cdots\right) .
\end{aligned}
$$

In particular for $x=1$ we have

$$
r \cdot(1 ; 1,0,0, \cdots)=\left(r ; f_{1}(r), f_{2}(r), \cdots\right) \text {. }
$$

Thus $(V, \cdot)$ is a $v$-hull of $G=\Sigma(\Gamma, R)$ and $G$ is also a vector lattice with respect to the natural scalar multiplication. Now $G \nsubseteq V$ since the maximal $l$-ideal of $V$ is laterally complete but the maximal $l$-ideal of $G$ is not.

Note, of course, that the $v$-hull $V$ of $G$ is not finite valued and it is not an a-extension of $G$. 


\section{Some open questions}

1) Does II always hold?

2) If $G$ is an archimedean $l$-group and each $G^{\gamma} / G_{\gamma} \cong R$ then does $G$ satisfy I?

3) If $G$ is an $l$-group and each $G(g)$ satisfies I then does $G$ satisfy I?

4) If $G$ is a vector lattice with a unique scalar multiplication then is $G$ archimedean?

\section{References}

S. Bernau (1965), 'Unique representations of Archimedean lattice groups and normal Archimedean lattice rings', Proc. London Math. Soc. 15, 599-631.

S. Bernau (1966), Orthocompletions of lattice groups', Proc. London Math. Soc. 16, 107-130.

A. Bigard and K. Keimel (1969), 'Sur les endomorphismes conservant les polares d'un groupe réticule archimedien', Bull. Soc. France 97, 381-398.

R. Bleier (1971), 'Minimal vector lattice cover', Bull. Australian Math. Soc. 5, 331-335.

R. Byrd (1966), Lattices ordered groups (Dissertation Tulane Univ. 1966).

Paul Conrad (1966), 'Archimedean extensions of lattice-ordered groups', J. Indian Math. Soc. 30, 131-160.

Paul Conrad, J. Harvey and C. Holland (1963), 'The Hahn embedding theorem for abelian lattice ordered groups', Trans. Amer. Math. Soc. 108, 143-169.

Paul Conrad (1970), Lattice ordered groups (Lecture Notes, Tulane Univ. 1970).

Paul Conrad and J. Diem (1971), 'The ring of polar preserving endomorphisms of an $l$-group', Illinois J. Math. 15, 222-240.

Paul Conrad (1974), 'Countable vector lattices', Bull. Austral. Math. Soc. 10, 371-376.

H. Hahn (1907), 'Uber die nichtarchimedischen Grossensysteme', Sitzungsberichte der Kaiserlchen Akademie der Wissensschaften, Vienna 116, 601-653.

K. Iwasawa (1943), 'On the structure of conditionally complete lattice-groups', Japan J. Math. 18, 777-789.

S. Wolfenstein, Contribution à l'étude des groupes réticules: extensions archimediennes, groupes à valeur normales (Thèse, $U$. of Paris).

University of Kansas

LAWRENCE

Kansas 6604, U.S.A. 